\title{
Numerical and theoretical study of a monodisperse hard-sphere glass former
}

\author{
P. Charbonneau, ${ }^{1}$ A. Ikeda, ${ }^{2}$ J. A. van Meel, ${ }^{3}$ and K. Miyazaki ${ }^{2}$ \\ ${ }^{1}$ Department of Chemistry, Duke University, Durham, North Carolina 27708, USA \\ ${ }^{2}$ Institute of Physics, University of Tsukuba, Tennodai 1-1-1, Tsukuba 305-8571, Japan \\ ${ }^{3}$ FOM Institute for Atomic and Molecular Physics, Science Park 113, 1098 XG Amsterdam, The Netherlands \\ (Received 8 September 2009; revised manuscript received 13 November 2009; published 14 April 2010)
}

\begin{abstract}
There exists a variety of theories of the glass transition and many more numerical models. But because the models need built-in complexity to prevent crystallization, comparisons with theory can be difficult. We study the dynamics of a deeply supersaturated monodisperse four-dimensional (4D) hard-sphere fluid, which has no such complexity, but whose strong intrinsic geometrical frustration inhibits crystallization, even when deeply supersaturated. As an application, we compare its behavior to the mode-coupling theory (MCT) of glass formation. We find MCT to describe this system better than any other structural glass formers in lower dimensions. The reduction in dynamical heterogeneity in 4D suggested by a milder violation of the StokesEinstein relation could explain the agreement. These results are consistent with a mean-field scenario of the glass transition.
\end{abstract}

DOI: 10.1103/PhysRevE.81.040501

PACS number(s): 64.70.qj, 61.43.Fs, 64.70.pm, 66.30.hh

Though the conversation started generations ago, scientists still debate the nature of the glass transition [1]. The multiplicity of competing frameworks even led some to declare that glass theories are more numerous than glass theorists [2]. To be fair, a great deal of progress toward a microscopic description has been made over the last couple of decades, but many fundamental questions remain unanswered. The intrinsic complexity of many glass-forming systems hinders progress. To avoid interference from precipitous crystallization, simulated fluids require built-in complexity: bidisperse $[3,4]$, polydisperse [5] or multicomponent [6] mixtures, anisotropic [7] or frustrated [8] pair interactions, etc. The situation is even more intricate in experiments, where good glass formers are, with only one reported exception [9], molecular liquids, polymer melts, or metallic alloys. Quantitative comparisons between the microscopic theories and these systems are formidably challenging, which impedes assessing the validity and limitations of the predictions [10]. A plain glass former, i.e., a monatomic one-component liquid with an interaction potential as simple as possible, would thus be greatly beneficial for the field's progress.

In this Rapid Communication, we report numerical simulations of a surprising glass former made of monodisperse four-dimensional (4D) hard spheres (HSs). Monodisperse HS have a single nontrivial thermodynamic parameter, the volume fraction $\eta$, which makes them the simplest model of isotropic fluids and crystals. Unlike in two-dimensional (2D) and three-dimensional (3D), monodisperse $\mathrm{HS}$ in 4D and higher dimensions are strongly geometrically frustrated with respect to the crystal [11]. The simplex-based liquid structure is geometrically distinct from the crystalline order, which inhibits nucleation and facilitates glass formation $[12,13]$. Compression studies of 4D HS suggest that the structural relaxation-time scale becomes longer than the slowest accessible quenching rate around $\eta \approx 0.40-0.41[12,14]$. A rough estimate gives the nucleation rate of reasonable system sizes for simulations to be eight to ten orders of magnitude slower than the structural relaxation-time scale $[13,15]$. In monodisperse $3 \mathrm{D} \mathrm{HS}$, in contrast, at $\eta \approx 0.54$ multiple crystal nuclei form simultaneously on the structural relaxation-time scale, while the onset of slow dynamics is generally agreed to be $\eta \approx 0.58$. Bidisperse or polydisperse HS are thus used to study glass formation (e.g., [4,5]). We perform moleculardynamics simulations with the event-driven package of Ref. [12] in a system of $N=4096$ particles, in order to examine the 4D system's glass-forming properties [16]. We expect finite-size effects to be small in this regime, as is the case at similar supersaturations in 3D for $N=512$ [17]. The structure factor $S(k)$ of the deeply supersaturated fluid remains liquid like at all densities, but the complete absence of crystallinity is also checked by an order parameter developed to detect nucleation [13]. Dynamically, the two-step growth of the mean-square displacement (MSD) $\left\langle\left|\mathbf{r}_{i}(t)-\mathbf{r}_{i}(0)\right|^{2}\right\rangle$, where $\mathbf{r}_{i}(t)$ is the position of the $i$ th particle, shows a lengthening caging plateau with density, a signature of structural glass formers (Fig. 1).

As an application of this simple model glass former, we consider the role of dimensionality in glass formation, which is a subject of considerable theoretical discussion $[14,18,19]$. The abrupt dynamical slowdown near the glass transition suggests the presence of a kinetic and/or a hidden thermodynamic singularity. The most direct evidence for such a singularity is the growth of spatiotemporal fluctuations on the structural relaxation-time scale, which results in fast and slow moving regions in supercooled liquids. This dynamical heterogeneity is typically monitored through four-point correlation functions [20] and Stokes-Einstein (SE) relationship violations $[1,21]$. As for conventional continuous phase transitions, the impact of these fluctuations should be reduced in higher dimensions as the system becomes more mean-fieldlike. Comparing systems of different dimensionality should allow to better understand the glass transition as a critical phenomenon and to test this mean-field scenario. A first attempt in this direction was recently made by Eaves and Reichman for a 4D binary Lennard-Jones (BLJ) model system [22], but the complication of identifying the dimensional correspondence between BLJ systems makes quantitative comparisons difficult.

Insights into the mean-field scenario of the glass transition 

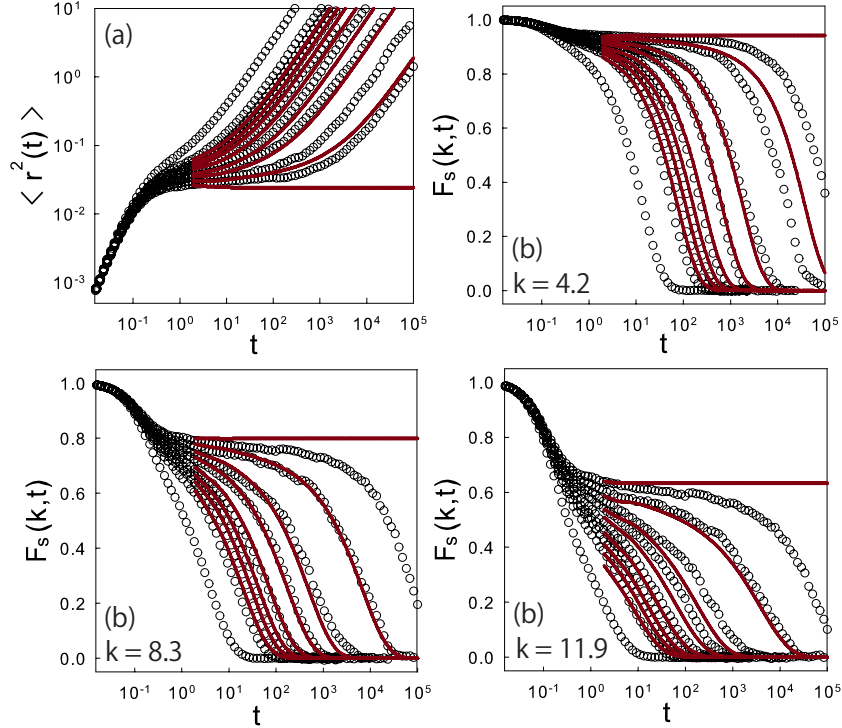

FIG. 1. (Color online) (a) MSD and (b) $F_{s}(k, t)$ at $\eta=0.370$, $0.386,0.389,0.392,0.395,0.398,0.401,0.404$, and 0.407 , from left to right. Solid lines are MCT fits using $\varepsilon$ as control parameter (see text). At the highest density $\tau_{\alpha}$ deviates from the power-law scaling of Fig. 2.

are gained by a mode-coupling theory (MCT) analysis of the dynamical results. A mean-field description of the thermodynamic "ideal" glass transition based on the replica theory suggests that HS undergo a dynamical transition at $\eta_{c}$, before reaching the thermodynamic glass transition of the one-step replica symmetry breaking at $\eta_{K}>\eta_{c}[14,23]$. This decoupling between dynamical and thermodynamic anomalies is demonstrated by simulation [24]. MCT is conjectured to be the dynamical counterpart of this approach below $\eta_{c}$, because its mathematical structure is equivalent to the dynamical equations of a mean-field $p$-spin-glass model [25] for which the relation between the dynamical and the thermodynamic glass transitions is rigorously established [26]. Though contentious, MCT is one of the most successful theories of the glass transition. It uses static structural information, such as the radial distribution function $g(r)$, to provide firstprinciples predictions of the slow dynamics of fluids before their dynamical arrest [27]. At mild supercooling it qualitatively captures the onset of the two-step decay of timecorrelation functions and the algebraic relaxation of the intermediate time regime. On approaching $\eta_{c}$ it predicts a power-law divergence of the structural relaxation time instead of the well-known Vogel-Fulcher-Tammann behavior [1]. This power law describes simulation and experimental observations fairly well for a range of densities below the $\eta_{c}$ fitted from the dynamical data [27], but the divergence at $\eta_{c}$ is rounded off due to activated events, which the theory does not capture. A failing of MCT is that it foretells a nonergodic freezing of the dynamics at a much lower $\eta_{c}$ (or higher temperature $T_{c}$ for thermal systems) than the experimental and simulation glass transition point $\eta_{g}$ (or $T_{g}$ ) [1]. Moreover the nonergodic freezing point obtained by fitting the simulation data with the MCT power law are systematically lower than what the theoretical predicts [28,29]. MCT also lacks an explanation for the violation of the SE relation, which is mostly attributed to strong dynamical heterogeneity near the glass transition and is missing in the theory [30]. Yet if MCT is indeed a dynamical mean-field theory, a reduction in the degree of heterogeneous dynamics by increasing dimensionality should improve the agreement with simulation results.

MCT is expressed as a series of nonlinear integrodifferential equations for correlation functions such as the intermediate scattering function $F(k, t)=N^{-1}\left\langle\delta \rho_{\mathbf{k}}(t) \delta \rho_{\mathbf{k}}^{*}(0)\right\rangle$, where $\delta \rho_{\mathbf{k}}(t)$ is the density fluctuation in reciprocal space. Generalization of the theory to 4D straightforwardly gives [31]

$$
\ddot{F}(k, t)+\Omega_{k}^{2} F(k, t)+\int_{0}^{t} d s M(k, t-s) \dot{F}(k, s)=0,
$$

where $\Omega_{k}^{2} \equiv k_{B} T k^{2} / m S(k), S(k) \equiv F(k, t=0)$ is the static structure factor, and $M(k, t)$ is the memory kernel. $M(k, t)$ can be further decomposed into fast and slow components $M(k, t)$ $=M_{\mathrm{fast}}(k, t)+M_{\mathrm{MCT}}(k, t)$ with

$$
M_{\mathrm{MCT}}(k, t)=\int_{0}^{\infty} d q \int_{|q-k|}^{|q+k|} d p V_{k}(q, p) F(q, t) F(p, t),
$$

where $V_{k}(q, p) \equiv \eta \sqrt{4 k^{2} p^{2}-f_{+}^{2}}\left[f_{+} c(q)+f_{-} c(p)\right]^{2} / 16 \pi k^{4}, c(k)$ $\equiv\{1-1 / S(k)\} / \rho$ is the direct correlation function, $\rho=N / V$ is the number density, and $f_{ \pm} \equiv k^{2} \pm\left(q^{2}-p^{2}\right)$. Binary collisions dominate the fast decaying part of the memory kernel $M_{\text {fast }}(k, t)$, which is conventionally determined by fitting simulation data [28]. Here, the analysis is done for the self part of the intermediate scattering function $F_{s}(k, t)$ $\equiv\left\langle e^{i \mathbf{k} \cdot\left[\mathbf{r}_{i}(t)-\mathbf{r}_{i}(0)\right]}\right\rangle$ for which the MCT expression is similar to Eq. (2) [31]. Because the MCT analysis is very sensitive to the details of the $S(k)$ input, particular care is taken to interpolate and extrapolate (to larger $k$ ) the simulation data to a continuous functional form. We extend the simulated $g(r)$ for distances larger than half the simulation box with a damped oscillatory function. The Fourier transform of $g(r)$ is in good agreement with the direct computation of $S(k)$. We compute (see Fig. 1) $F_{s}(k, t)$ for several wave vectors, one of which $(k=8.3)$ is close to the first peak of the structure factor and thus not too far from the dominant microscopic caging and relaxation length scales [32]. The decorrelation of density fluctuations displays the characteristic caging plateau and allows for the extraction of the structural relaxation time $\tau_{\alpha}$, defined as $F_{s}\left(k, \tau_{\alpha}\right)=1 / e$.

MCT predicts [Eq. (1)] that both $\tau_{\alpha}$ and the diffusion coefficient $D$ scale with the same power-law exponent $\gamma$ and critical density $\eta_{c}$, i.e., $\tau_{\alpha}^{-1}, D \propto\left|\eta_{c}-\eta\right|^{\gamma}$, with $\gamma^{\mathrm{MCT}}=2.8$ and $\eta_{c}^{\mathrm{MCT}}=0.379$. In Fig. 2, we fit the simulation data to this form by fixing $\gamma^{\mathrm{MCT}}$ and leaving $\eta_{c}$ free. All the data except for the densest system follow a power law with $\eta_{c, D}^{\text {sim }}=0.407$ and $\eta_{c, \tau}^{\text {sim }}=0.406$ for all wave vectors. The discrepancy between $\eta_{c, D}^{\text {sim }}$ and $\eta_{c, \tau_{\alpha}}^{\text {sim }}$ is smaller $(0.2 \%)$ than for 3D HS $(0.5 \%)$ [4], and the concordance with $\eta_{c}^{\mathrm{MCT}}$ slightly improves. It is interesting to note that $\eta_{c}^{\text {sim }}$ is very close to the extrapolated dynamical arrest point independently obtained at very slow compression rates $[12,14]$. For consistency check, we also fit $\gamma$ while fixing $\eta_{c}^{\text {sim }}=0.406$, which gives $\gamma_{D}^{\text {sim }}=2.4$ and $\gamma_{\tau_{\alpha}}^{\text {sim }}$ $=2.7$ for all wave vectors. In contrast, for 3D BLJ [6,33], which is the best characterized system, $\gamma_{D}^{\mathrm{sim}} \approx 1.8$ and $\gamma_{\tau_{\alpha}}^{\mathrm{sim}}$ 


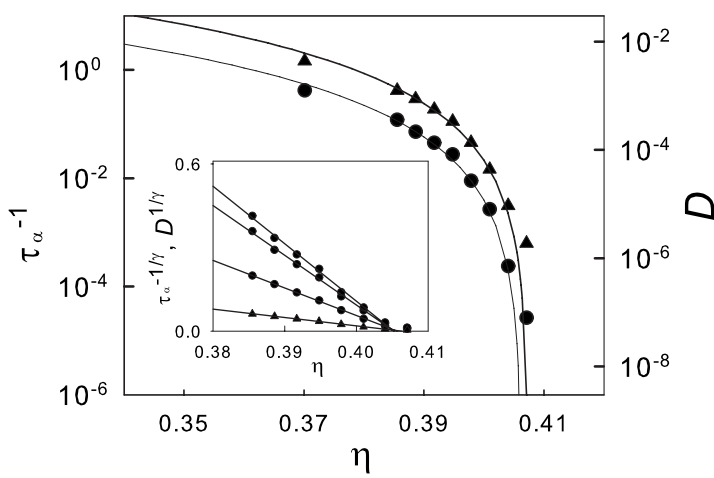

FIG. 2. Power-law fit of $D(\mathbf{\Delta})$ and $\tau_{\alpha}^{-1}$ for $k=8.3(\mathbf{O})$. Inset: $D^{1 / \gamma}$ and $\tau_{\alpha}^{-1 / \gamma}$ for $k=8.3,7.5$, and 4.2 , from top to bottom, scale linearly with a shared intersection point.

$\approx 2.3$, whereas $\gamma^{\mathrm{MCT}}=2.46$. Another notable feature is that the power law fits over almost four time decades, reaching densities where $\varepsilon \equiv 1-\eta / \eta_{c}^{\text {sim }}$ is less than $0.5 \%$ (Fig. 2). In 2D and 3D systems the structural relaxation-time scale follow a power law for only two or at most three decades [5,6,33-35], before the theoretical description breaks down because of activated processes.

We also consider how well MCT describes the full MSD and $F_{s}(k, t)$ curves, using $\varepsilon$ as input parameter to scale out the $\eta_{c}$ dependence (Fig. 1). To tease out the long-time MCT behavior from Eq. (1), the short-time $F_{s}(k, t)$ decay (up to $t$ $\approx 10^{\circ}$ ) is imported from the simulation curves. This procedure is equivalent to, but more direct than the standard fit of $M_{\text {fast }}(k, t)$ from simulation [28]. The concordance between simulation and calculated $F_{s}(k, t)$ is almost perfect for $k$ $=8.3$ and 11.9, up to densities where $\tau_{\alpha}$ deviates from the power-law behavior and MCT foretells the nonergodic transition. The calculated MSD also matches the simulated curves quite well, except for $\eta=0.404$ and above. The correspondence between simulation and MCT is noticeably better than for lower-dimensional systems, where for every relaxation curve both $\eta_{c}$ and $k$ must be rescaled, in order to achieve a reasonable collapse [36]. Besides the power-law scaling, MCT successfully describes several other features in $3 \mathrm{D}$, such as the time-temperature superposition, the vonSchweidler law at the beta relaxation regimes, the $k$-dependence of the plateau height (the nonergodic parameter), the exponents of stretched exponential relaxation at the alpha relaxation regime $\beta(k)$, and the beta relaxation exponent $b$ [27]. The concordance between theory and simulation for all of these features is at least as good for 4D HS as for 3D systems.

The quantitative improvement of MCT predictions in 4D over 3D suggests that fluctuations are less important in higher dimensions. We analyze the system's dynamical heterogeneity through the SE relation to verify this hypothesis. Figure 3 shows $D \tau_{\alpha}$ for the full range of densities explored along with the corresponding 3D HS results [4,5]. In order to put the different dimensions on an equal footing, we plot $D \tau_{\alpha}$ against the diffusion coefficient $D$. The SE relation holds for diffusivities over a decade smaller in 4D than in 3D (up to $\varepsilon \approx 1 \%$ ), which is slightly before $D$ and $\tau_{\alpha}$ start deviating from the MCT-predicted power law in Fig. 2. If a similar

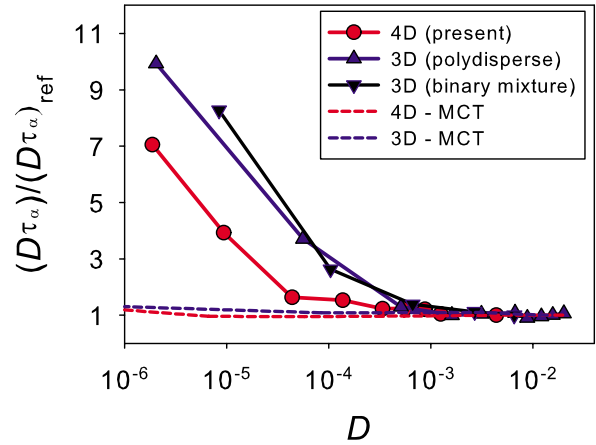

FIG. 3. (Color online) Violation of the SE relation in 4D monodisperse and 3D bidisperse [4] and polydisperse [5] HS, where $D \tau_{\alpha}$ is normalized by its low-density $\left(D \tau_{\alpha}\right)_{\text {ref }}$ value. MCT predicts only a very small violation of the SE relation for both $3 \mathrm{D}$ and $4 \mathrm{D}$ systems.

degree of polydispersity were used in $4 \mathrm{D}$ as in $3 \mathrm{D}$ we expect the SE violation to be suppressed even more [37]. The suppression of the SE relation violation and the better agreement of all available dynamical observables with MCT predictions suggest that 4D HS are dynamically more mean-field-like than the $2 \mathrm{D}$ and $3 \mathrm{D}$ equivalents. The improvement of the agreement with MCT is however incremental, which hints that if an upper critical dimension $d_{c}$ for the glass transition exists, it is larger than four. This result is consistent with general theoretical arguments that give $d_{c}=8[18,38]$. Biroli et al. also obtained that the SE relation violation should scale as $D \tau_{\alpha} \sim \varepsilon^{d / 4-2}$ [18]. The growth in Fig. 3 is not inconsistent with this scaling, but the SE relation violation for the range of densities we explore is too mild to be conclusive about the exponent. A more direct measure of dynamical heterogeneity would be to compute the four-point correlation function $\chi_{4}(t)$, but the lack of statistical accuracy and the relatively small system sizes prevent us from reporting the results here. We will consider $\chi_{4}(t)$ in future studies.

The 4D monodisperse HS fluid we study is quite convenient to examine the glass transition. Its simplicity and slow nucleation rate allow high-accuracy comparisons of its glassforming properties with microscopic theories. The agreement of the system with MCT, which is broader than for any lower-dimensional equivalents, and the strong suppression of SE relation violation are consistent with the dynamical mean-field scenario of the glass transition [14]. The results also suggest that $4 \mathrm{D}$ is still below the upper critical dimension, if it exists, because the fluctuations due to activated processes round off the sharp dynamical singularity. Given that even the mean-field picture has not been rigorously established for the structural glass transition, the study of clean, higher-dimensional systems such as 4D HS is likely to play a crucial role in assessing the validity and limitations of the various glass theories.

We thank D. Reichman and F. Zamponi for stimulating discussions. P.C. acknowledges computer time at the Dutch center for high-performance computing SARA and startup funding from Duke University. This work is partially supported by Grant-in-Aid for JSPS Foundation (A.I.), KAKENHI Grant No. 21540416, (K.M.), and Priority Areas "Soft Matter Physics" (K.M.). 
[1] K. Binder and W. Kob, Glassy Materials and Disordered Solids (World Scientific, Singapore, 2005).

[2] K. Chang, The New York Times, July 29, 2008.

[3] B. Bernu, Y. Hiwatari, and J. P. Hansen, J. Phys. C 18, L371 (1985).

[4] G. Foffi, W. Götze, F. Sciortino, P. Tartaglia, and T. Voigtmann, Phys. Rev. E 69, 011505 (2004).

[5] S. K. Kumar, G. Szamel, and J. F. Douglas, J. Chem. Phys. 124, 214501 (2006).

[6] W. Kob and H. C. Andersen, Phys. Rev. Lett. 73, 1376 (1994).

[7] H. Shintani and H. Tanaka, Nat. Phys. 2, 200 (2006).

[8] M. Dzugutov, S. I. Simdyankin, and F. H. M. Zetterling, Phys. Rev. Lett. 89, 195701 (2002).

[9] M. H. Bhat, V. Molinero, E. Soignard, V. C. Solomon, S. Sastry, J. L. Yarger, and C. A. Angell, Nature (London) 448, 787 (2007).

[10] A. Cavagna, Phys. Rep. 476, 51 (2009).

[11] J. A. van Meel, B. Charbonneau, A. Fortini, and P. Charbonneau, Phys. Rev. E 80, 061110 (2009).

[12] M. Skoge, A. Donev, F. H. Stillinger, and S. Torquato, Phys. Rev. E 74, 041127 (2006).

[13] J. A. van Meel, D. Frenkel, and P. Charbonneau, Phys. Rev. E 79, 030201(R) (2009).

[14] G. Parisi and F. Zamponi, Rev. Mod. Phys. 82, 789 (2010).

[15] S. Auer and D. Frenkel, Nature (London) 409, 1020 (2001).

[16] The particle diameter $\sigma$ sets the unit of length, the thermal energy $k_{B} T$ the energy unit, $m$ the unit of mass, and time $t$ is expressed in $\left(k_{B} T / m \sigma^{2}\right)^{1 / 2}$ units. At least eight independent initial configurations are equilibrated after a slow compression from the ideal-gas limit.

[17] S. Karmakar, C. Dasgupta, and S. Sastry, Proc. Natl. Acad. Sci. U.S.A. 106, 3675 (2009).

[18] G. Biroli and J.-P. Bouchaud, J. Phys.: Condens. Matter 19, 205101 (2007); A. Andreanov, G. Biroli, and J.-P. Bouchaud, EPL 88, 16001 (2009).

[19] T. R. Kirkpatrick and P. G. Wolynes, Phys. Rev. A 35, 3072
(1987).

[20] C. Toninelli, M. Wyart, L. Berthier, G. Biroli, and J.-P. Bouchaud, Phys. Rev. E 71, 041505 (2005).

[21] M. T. Cicerone and M. D. Ediger, J. Chem. Phys. 104, 7210 (1996).

[22] J. D. Eaves and D. R. Reichman, Proc. Natl. Acad. Sci. U.S.A. 106, 15171 (2009).

[23] M. Mézard and G. Parisi, Phys. Rev. Lett. 82, 747 (1999).

[24] L. Santen and W. Krauth, Nature (London) 405, 550 (2000).

[25] T. R. Kirkpatrick and D. Thirumalai, Phys. Rev. Lett. 58, 2091 (1987).

[26] T. Castellani and A. Cavagna, J. Stat. Mech. (2005), P05012.

[27] W. Götze, Complex Dynamics of Glass-Forming Liquids (Oxford University Press, Oxford, 2009).

[28] W. Kob, M. Nauroth, and F. Sciortino, J. Non-Cryst. Solids 307-310, 181 (2002).

[29] Y. Brumer and D. R. Reichman, Phys. Rev. E 69, 041202 (2004).

[30] P. Mayer, K. Miyazaki, and D. R. Reichman, Phys. Rev. Lett. 97, 095702 (2006).

[31] M. Bayer, J. M. Brader, F. Ebert, M. Fuchs, E. Lange, G. Maret, R. Schilling, M. Sperl, and J. P. Wittmer, Phys. Rev. E 76, 011508 (2007).

[32] P. Charbonneau and D. R. Reichman, Phys. Rev. Lett. 99, 135701 (2007)

[33] E. Flenner and G. Szamel, Phys. Rev. E 72, 011205 (2005).

[34] G. Foffi, W. Götze, F. Sciortino, P. Tartaglia, and T. Voigtmann, Phys. Rev. Lett. 91, 085701 (2003).

[35] L. Berthier and T. Witten, Phys. Rev. E 80, 021502 (2009).

[36] T. Voigtmann, A. M. Puertas, and M. Fuchs, Phys. Rev. E 70, 061506 (2004)

[37] T. Kawasaki, T. Araki, and H. Tanaka, Phys. Rev. Lett. 99, 215701 (2007); S. E. Abraham, S. M. Bhattacharrya, and B. Bagchi, ibid. 100, 167801 (2008).

[38] G. Biroli, J.-P. Bouchaud, K. Miyazaki, and D. R. Reichman, Phys. Rev. Lett. 97, 195701 (2006). 